\title{
Correction to: Auxiliaries to Abusive Supervisors: The Spillover Effects of Peer Mistreatment on Employee Performance
}

\author{
Yuntao Bai ${ }^{1} \cdot$ Lili Lu $^{1} \cdot$ Li Lin-Schilstra ${ }^{2,3}$
}

Published online: 16 March 2021

(c) Springer Nature B.V. 2021

\section{Correction to: Journal of Business Ethics https://doi.org/10.1007/s10551-021-04768-6}

In the initial online publication the funding information was missing. The original article has been corrected.

Funding This research was supported by National Natural Science Foundation of China (ID: 71672155), Natural Science Foundation of Fujian Province (ID: 2020J01032) and Program for New Century Excellent Talents in China Fujian Province University for the first author.

Publisher's Note Springer Nature remains neutral with regard to jurisdictional claims in published maps and institutional affiliations.

The original article can be found online at https://doi.org/10.1007/ s10551-021-04768-6.

Li Lin-Schilstra

li.lin@njau.edu.cn

Yuntao Bai

ytbai@xmu.edu.cn

Lili Lu

jasminelu_8030@hotmail.com

1 School of Management, Xiamen University, 422 Siming S

Rd, Siming District, Xiamen 361005, Fujian, China

2 College of Economics and Management, Nanjing Agricultural University, Nanjing 210014, Jiangsu, China

3 Marketing and Consumer Behaviour Group, Wageningen University, Hollandseweg 1, 6706 KN Wageningen,

The Netherlands 